\title{
Functional Neuroimaging in Irritable Bowel Syndrome: A Systematic Review Highlights Common Brain Alterations With Functional Movement Disorders
}

\begin{abstract}
Veronica Nisticò, ${ }^{1,2,3 *}$ Roberta E Rossi, ${ }^{4,5}$ Andrea M D'Arrigo, ${ }^{6}$ Alberto Priori, ${ }^{1,2,7}$ Orsola Gambini, ${ }^{1,2,8}$ and Benedetta Demartini ${ }^{1,2,8}$
${ }^{\prime}$ Dipartimento di Scienze della Salute, Università degli Studi di Milano, Milan, Italy; “'Aldo Ravelli” Research Center for Neurotechnology and Experimental Brain Therapeutics, Università degli Studi di Milano, Milan, Italy; ${ }^{3}$ Department of Psychology, University of Milan-Bicocca, Milan, Italy; ${ }^{4}$ Gastro-intestinal Surgery and Liver Transplantation Unit, Fondazione IRCCS Istituto Nazionale dei Tumori, Milan, Italy; ${ }^{5}$ Department of Pathophysiology and Organ Transplant, Università degli Studi di Milano, Milan, Italy; ${ }^{6}$ Department of Neurology, ASST Fatebenefratelli Sacco, Ospedale Fatebenefratelli, Milan, Italy; ${ }^{7}$ III Clinica Neurologica, ASST Santi Paolo e Carlo, Presidio San Paolo, Milan, Italy; and ${ }^{8}$ Unità di Psichiatria 52, ASST Santi Paolo e Carlo, Presidio San Paolo, Milan, Italy
\end{abstract}

Irritable bowel syndrome (IBS) is a chronic functional gastrointestinal disorder characterized by recurring abdominal pain and altered bowel habits without detectable organic causes. This study aims to provide a comprehensive overview of the literature on functional neuroimaging in IBS and to highlight brain alterations similarities with other functional disorders - functional movement disorders in particular. We conducted the bibliographic search via PubMed in August 2020 and included 50 studies following Preferred Reporting Items for Systematic Reviews and Meta-analyses (PRISMA) guidelines for systematic reviews. Overall, our findings showed an aberrant activation and functional connectivity of the insular, cingulate, sensorimotor and frontal cortices, the amygdala and the hippocampus, suggesting an altered activity of the homeostatic and salience network and of the autonomous nervous system. Moreover, glutamatergic dysfunction in the anterior insula and hypothalamic pituitary axis dysregulation were often reported. These alterations seem to be very similar to those observed in patients with functional movement disorders. Hence, we speculate that different functional disturbances might share a common pathophysiology and we discussed our findings in the light of a Bayesian model framework.

(J Neurogastroenterol Motil 2022;28:185-203)

Key Words

Amygdala; Brain; Conversion disorders; Functional neuroimaging; Irritable bowel syndrome

\section{Introduction}

Irritable bowel syndrome (IBS) is a chronic functional gastro- intestinal disorder (FGID) characterized by recurring abdominal pain associated with altered bowel habits without detectable organic causes. ${ }^{1,2}$ The definition of IBS is based on the Rome IV criteria: recurrent abdominal pain at least 3 days per month in the last 3

\footnotetext{
Received: April 17, 2021 Revised: September 3, 2021 Accepted: November 24, 2021

(a) This is an Open Access article distributed under the terms of the Creative Commons Attribution Non-Commercial License (http://creativecommons. org/licenses/by-nc/4.0) which permits unrestricted non-commercial use, distribution, and reproduction in any medium, provided the original work is properly cited.

*Correspondence: Veronica Nisticò, MSc

Dipartimento di Scienze della Salute, Università degli Studi di Milano, via A. di Rudinì, 8, Milano, 20142, Italy

Tel: +39-02-81844730, Fax: +39-02-81844730, E-mail: veronica.nistico@unimi.it

Veronica Nisticò and Roberta E Rossi equally contributed to the study
} 
months, associated with a change in stool frequency or form and improvement with defecation. ${ }^{2}$ IBS is a multi-factorial disorder with a complex bio-psychosocial pathophysiology, ${ }^{3}$ including altered gastrointestinal motility, visceral hyperalgesia, ${ }^{4}$ increased intestinal permeability, immune activation, ${ }^{5}$ altered microbiota, ${ }^{6}$ and disrupted communication between the gastrointestinal tract and the central nervous system. ${ }^{7}$ The relevance of bidirectional communication pathways between the brain and the gastrointestinal system, namely the brain-gut-axis, is increasingly acknowledged as underlying pathophysiological mechanism of different somatic and neuropsychiatric disturbances. ${ }^{8}$ Multiple brain networks (salience, sensorimotor, and executive-control networks) have been reported to mediate the effects of affect, mood, and environmental factors on gut function and pain perception, resulting in visceral hypersensitivity and altered bowel habits ${ }^{9,10}$ and IBS can be considered an exemplary disorder of brain-gut communication. Therefore, in the recent years, several neuroimaging studies have been conducted to investigate brain alterations associated with IBS. Recent studies implementing quantitative magnetic resonance spectroscopy (qMRS) highlighted an aberrant glutamatergic neurotransmission in limbic structures of IBS patients. ${ }^{11-13}$ Bednarska et $\mathrm{al}^{11}$ observed that increased concentration of glutamate was also reported in the posterior insula ( $\mathrm{pINS}$ ) of patients with fibromyalgia. ${ }^{14}$ Moreover, according to a recent case-control study from our group, ${ }^{15}$ a glutamate increase has been detected in the limbic system of patients suffering from functional movement disorders (FMD, also called conversion disorders) when compared to healthy controls (HC). Based on these observations, herein we conducted a systematic review of the literature focused on functional neuroimaging in the IBS setting. Consequently, we discuss whether these shared brain alterations may represent a common pathophysiology for different functional disturbances.

\section{Methods}

We followed Preferred Reporting Items for Systematic Reviews and Meta-analyses (PRISMA) guidelines for systematic reviews. ${ }^{16}$ The bibliographic search was conducted on PubMed in August 2020. Moreover, we looked through reference lists of screened articles.

PRISMA 2009 flow diagram
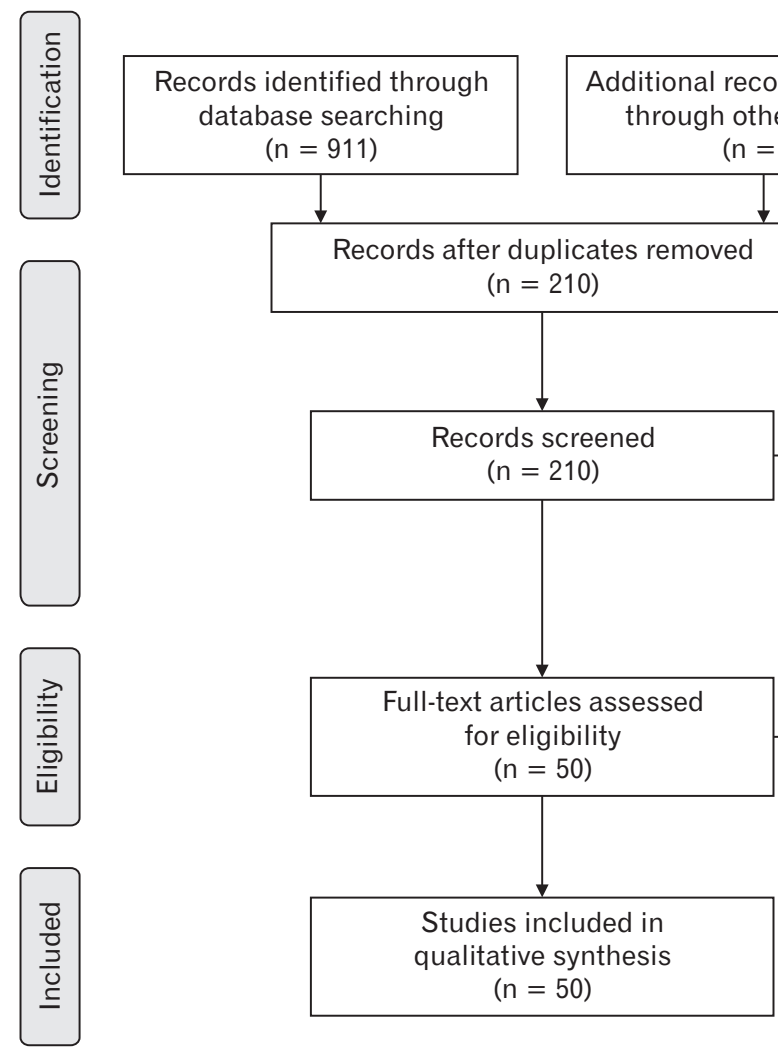

For more information, visit www.prisma-statement.org.
Additional records identified through other sources $(n=2)$ reasons: $n=160$ [n = 9: language;

$\mathrm{n}=67$ : review, commentaries or meta-analyses; $\mathrm{n}=13$ : no humans; $\mathrm{n}=22$ : no IBS;

$\mathrm{n}=11$ : no functional neuroimaging; $\mathrm{n}=21$ : no HC;

$\mathrm{n}=13$ : case series;

$\mathrm{n}=4$ : pediatric IBS]

Full-text articles excluded, with reasons $(\mathrm{n}=0)$
Figure 1. Preferred Reporting Items for Systematic Reviews and Meta-analyses (PRISMA) flow diagram illustrating the bibliographic search and the selection process. Adapted from Moher et al. ${ }^{16}$ IBS, irritable bowel syndrome; HC, healthy control. 
Inclusion criteria were: (1) English-written articles, (2) articles including only human subjects above 18 years old, (3) studies on IBS, and (4) functional neuroimaging studies.

Exclusion criteria were: (1) studies about any other functional somatic disorder, (2) review articles, (3) structural neuroimaging studies, (4) case series (less than 10 patients with IBS), (5) studies without a $\mathrm{HC}$ group, and (6) studies including pediatric patients with IBS.

The search was conducted using the following keywords: (irritable bowel syndrome), (spastic colon), (spastic bowel), (mucous colitis), and (nervous colon); and combined on a string with the keywords: (functional neuroimaging) and (functional brain imaging). Supplementary Table shows results for each string on PubMed. We retrieved 911 articles. After removing duplicates, 210 articles remained, which were independently screened by 2 authors (V.N. and R.E.R.) by reading abstracts and full text. Disagreement was resolved by discussion between the 2 independent authors; if no agreement was reached, a third independent party (B.D.) was involved as an arbiter. Fifty studies were selected (Fig. 1). Results are presented divided up by methodology in Table 1 and synthetically described in Table 2.

\section{Results}

\section{Functional Magnetic Resonance Imaging at Resting- state}

Compared to $\mathrm{HC}$, patients with IBS showed hypoactivity at resting-state in: (1) the left superior frontal gyrus (SFG), the right hippocampus, the bilateral post-central gyrus (primary somatosensory cortex $[\mathrm{S} 1])$, and the right superior temporal pole ${ }^{17} ;(2)$ the right middle frontal gyrus $(\mathrm{MFG})^{17,18}$ and the right orbital part of the $\mathrm{SFG}^{18}$; (3) several default mode network (DMN) regions: the medial prefrontal cortex ( $\mathrm{mPFC})$, the posterior cingulate cortex (PCC), and the bilateral inferior parietal cortices. ${ }^{18}$ Hyperactivity was found in the left median cingulate cortex (MCC), ${ }^{17}$ the calcarine, ${ }^{17}$ the bilateral pINS, ${ }^{18}$ and cuneus. ${ }^{18}$ Duration of the IBS disease positively correlated with the right $\mathrm{MFG}$ activity and negatively correlated with the left MCC activity ${ }^{17}$; dorsal and ventral anterior cingulate cortex (ACC) hypoactivity disappeared after controlling for anxiety and depression, leading the authors to hypothesize that high anxious-depressive symptomatology may explain the intrinsic decreased brain activity in regions involved in affective processing (the ACC). ${ }^{18}$ Focusing on gender- and disease-related differences, Hong and colleagues ${ }^{19}$ found that: (1) female IBS pa- tients, compared to $\mathrm{HC}$, showed hyperactivity in the amygdala and hippocampus, and hypoactivity in sensorimotor regions; (2) both female $\mathrm{HC}$ and female IBS patients showed higher activity in the amygdala and hippocampus than male $\mathrm{HC}$ and male IBS patients, respectively; female IBS patients, compared with males, showed insular hyperactivity; and (3) in female patients, altered activity correlated with abdominal discomfort ratings. ${ }^{19}$

In summary, these studies show a global frontal, sensorimotor and DMN hypoactivity, and a cingulate, insular and amygdala hyperactivity in patients with IBS at resting state, compared to $\mathrm{HC}$.

\section{Resting-state functional connectivity}

In IBS patients, increased connectivity was found between: (1) the left MCC and left SFG; ${ }^{17}$ (2) the right MFG, left SFG, and left $\mathrm{PCC}^{17}$; and (3) the mPFC and cuneus (negative $\mathrm{FC}^{18}$ ). Decreased connectivity was found between: (1) the right superior parietal gyrus and left rectus ${ }^{17},(2) \mathrm{mPFC}$ and the right orbital part of the SFG (positive connectivity ${ }^{18}$ ), (3) ventral ACC and PCC (positive connectivity ${ }^{18}$ ), and (4) mPFC and left pINS (negative connectivity $^{18}$ ). Qi and colleagues ${ }^{18}$ found that $\mathrm{FC}$ was not influenced by anxiety and depression, although other studies did not confirm this finding. ${ }^{20,21}$ Moreover, Qi and colleagues ${ }^{21}$ showed that IBS patients, compared to $\mathrm{HC}$, had higher interhemispheric FC between thalamus (bilateral), PCC, cuneus, lingual gyri, and cerebellar lobes, together with lower interhemispheric FC between bilateral ventral ACC and inferior parietal lobules. Controlling for anxiety and depression, connectivity differences in the ventral ACC were abolished. Weng and colleagues ${ }^{22}$ calculated the long-range and short-range FC density (LR- and SR-FCD: the amount of distant and local functional connections of cortical areas). Compared to HC, IBS patients showed several FCD alteration in areas deputed to sensorimotor, homeostatic, emotional and cognitive regulation: (1) decreased LR- and SR-FCD in bilateral anterior MCC and inferior parietal lobules; (2) decreased LR-FCD in the right anterior insula (aINS), positively correlated with severity of IBS symptoms; (3) decreased SR-FCD in bilateral PFC, subgenual ACC and caudate, positively correlated with disease duration; (4) increased LRand SR-FCD in S1; (5) increased LR-FCD in right supplementary motor area (SMA); and (6) increased SR-FCD in occipital lobe. Moreover, they found altered FC in IBS patients' pain matrix, in particular: increased $\mathrm{FC}$ between the anterior MCC, right aINS, and superior prefrontal cortices; decreased $\mathrm{FC}$ between anterior $\mathrm{MCC}$, right aINS, and PCC/precuneus. Postcentral cortices with stronger FCD showed: (1) increased FC with bilateral pre-central cortices, PCC/precuneus and $\mathrm{mPFC}$; and (2) decreased FC 


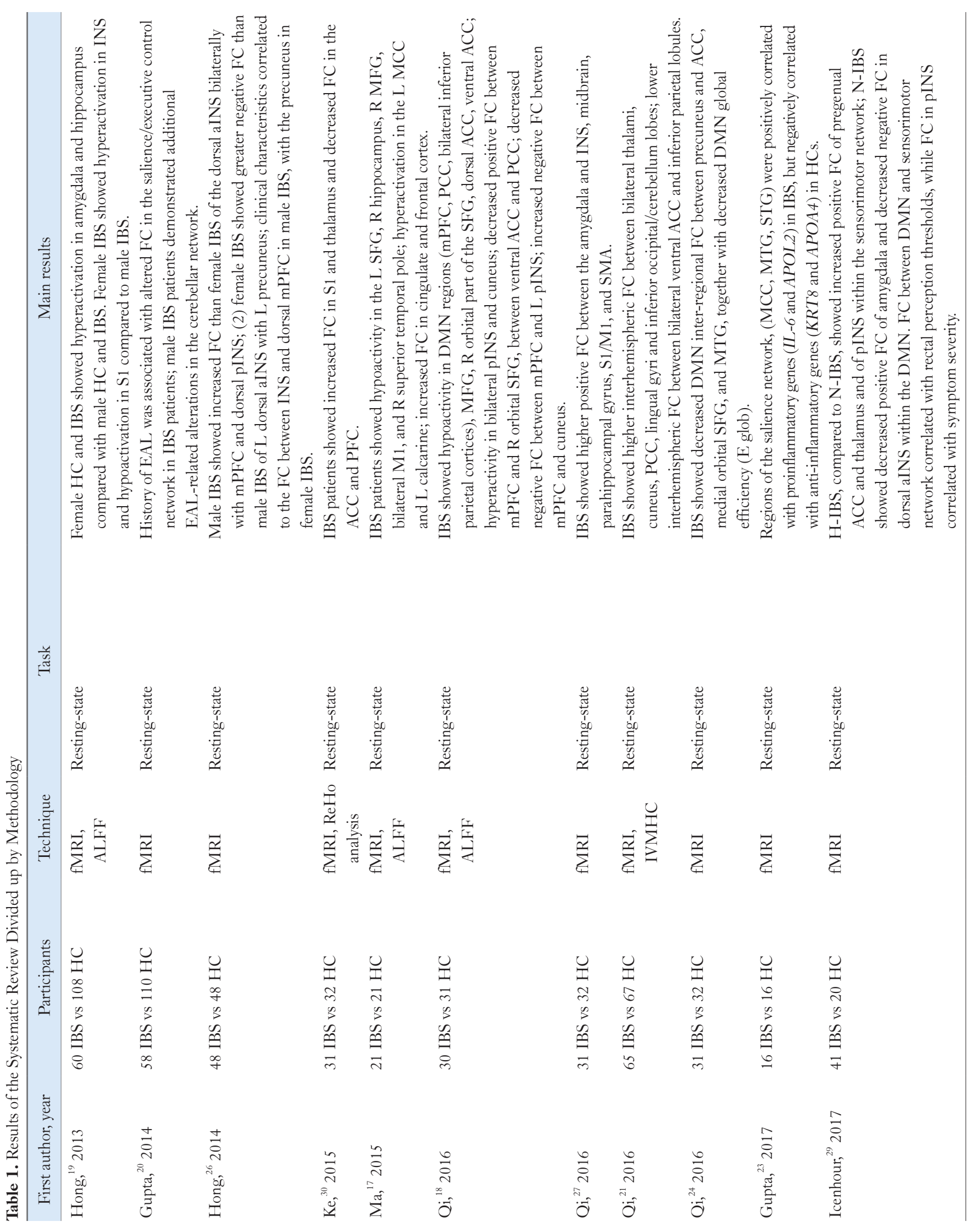




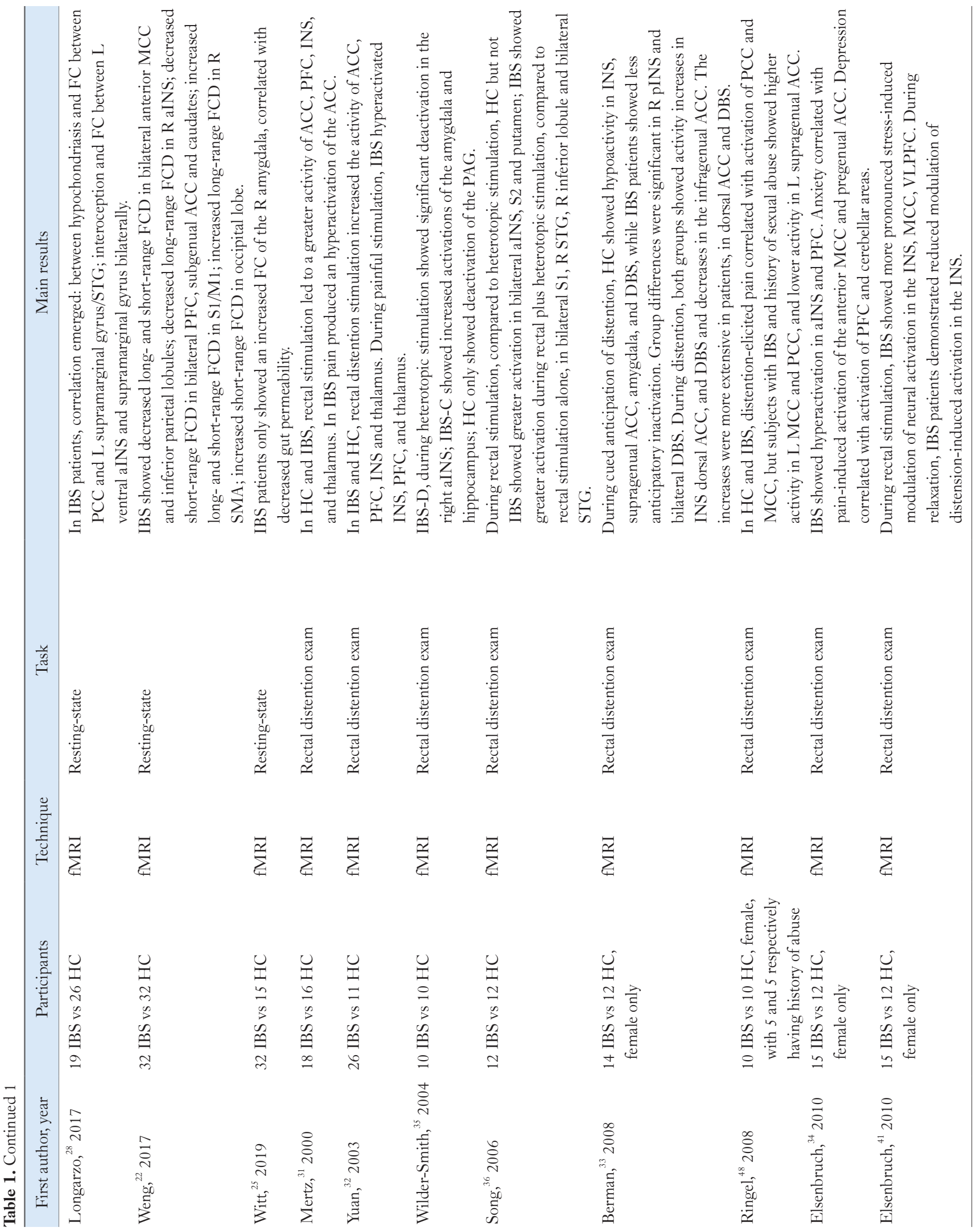




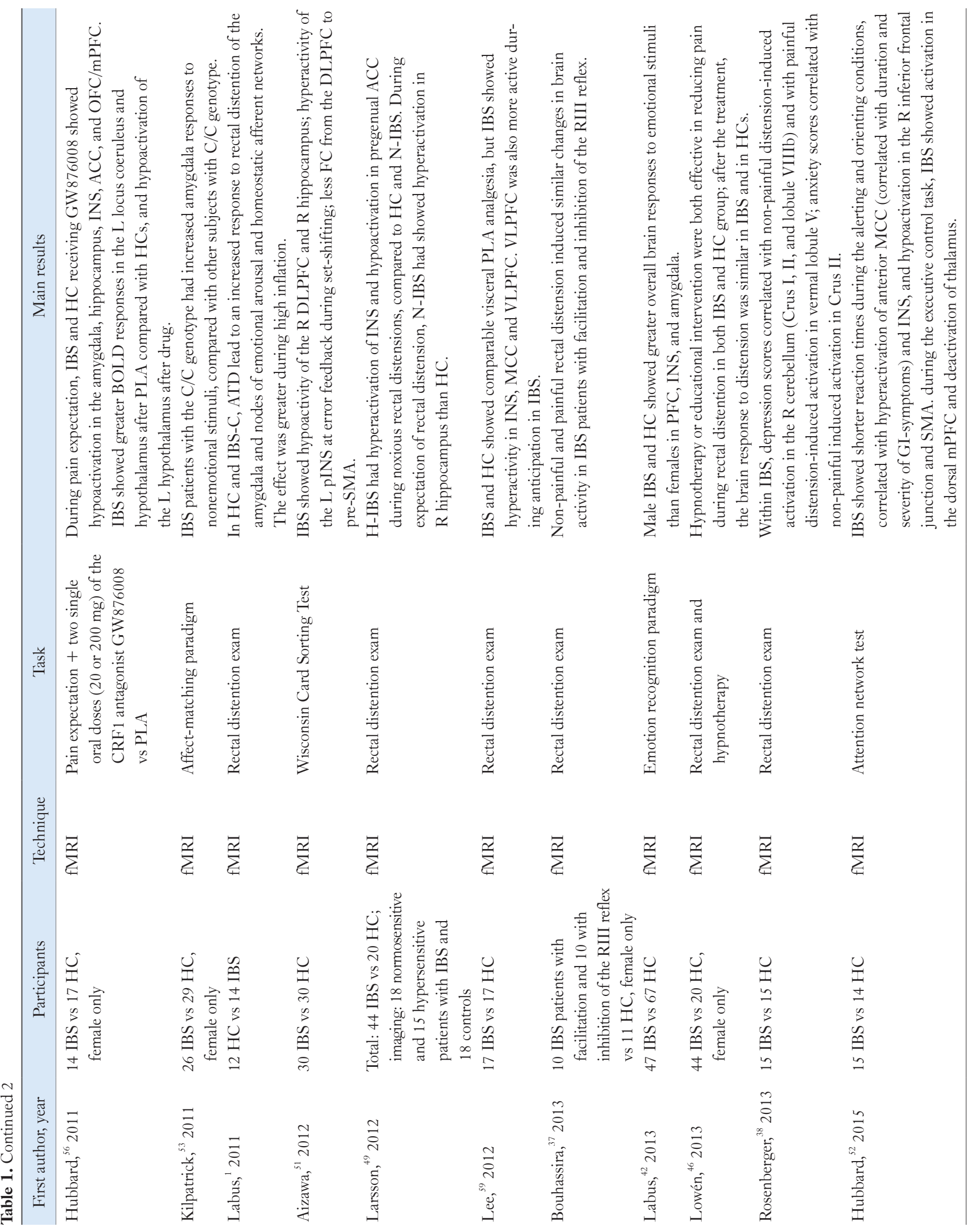




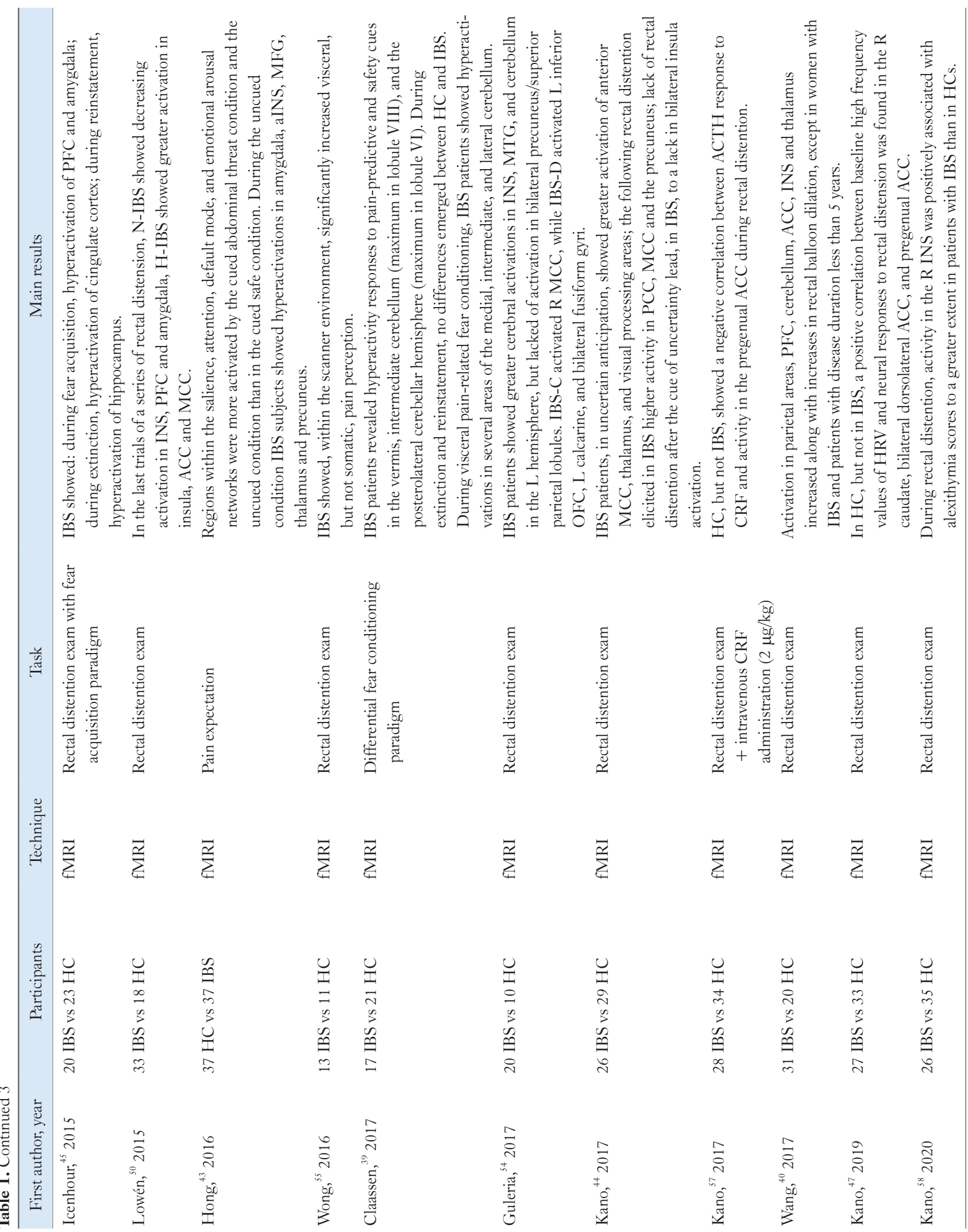




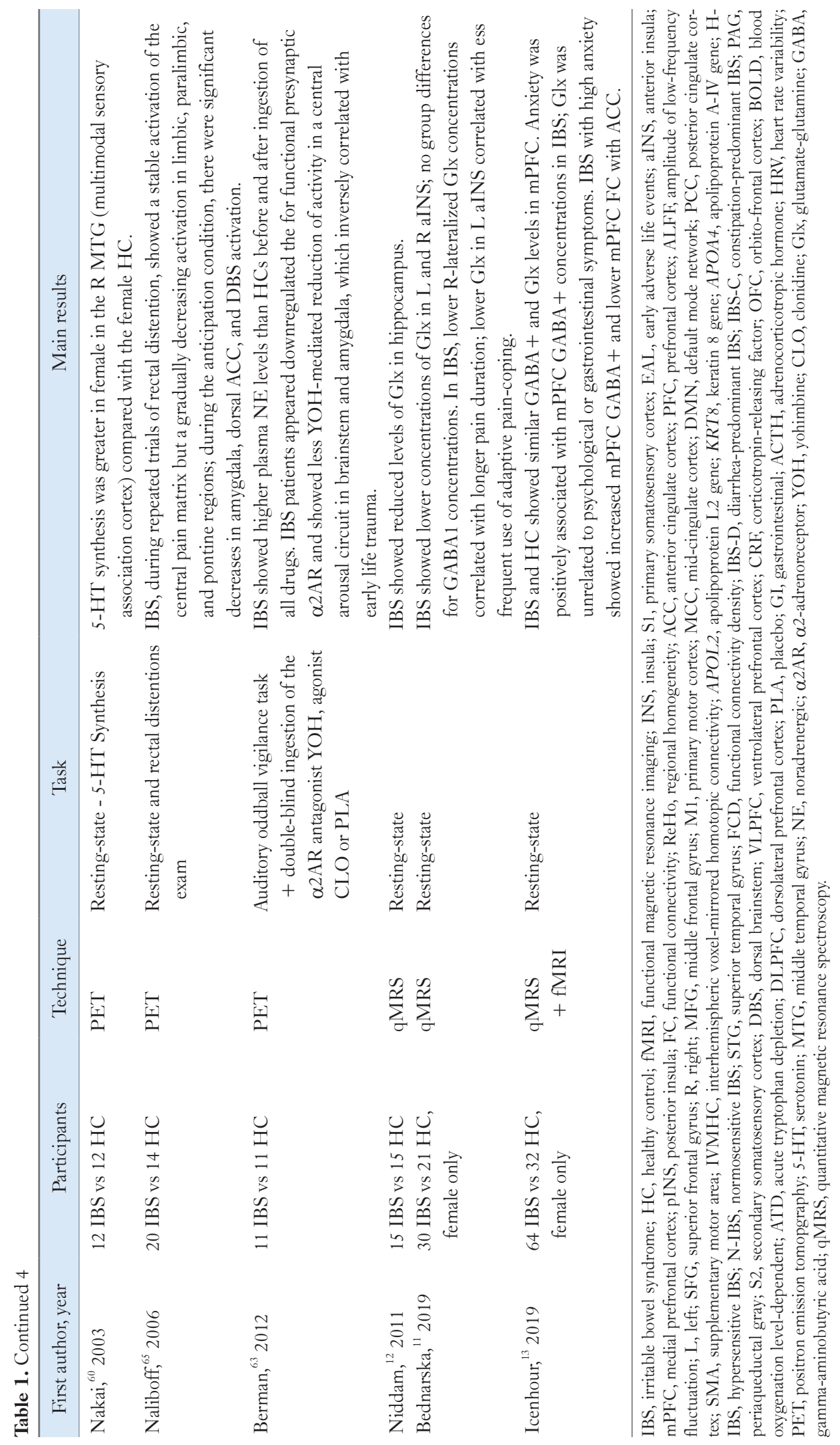


Table 2. Main Findings of the Systematic Review Divided up by Brain Areas

\begin{tabular}{|c|c|}
\hline Network & Main findings \\
\hline Sensorimotor network & $\begin{array}{l}\text { Resting state: } \mathrm{S} 1 \text { hypoactivation, M1 hyperactivation, increased FC between } \mathrm{S} 1 \text { and thalamus, and aberrant FC between } \\
\text { the sensorimotor network and affective-interoceptive areas (amygdala and insula in particular). H-IBS showed } \\
\text { increased FC between sensorimotor network and pINS, compared to N-IBS. }\end{array}$ \\
\hline \multirow[t]{3}{*}{$\begin{array}{l}\text { Affective and } \\
\text { interoceptive areas }\end{array}$} & $\begin{array}{l}\text { Resting state: aberrant FC between the cingulate and the frontal cortices, and between amygdala, insula, sensorimotor } \\
\text { network, and hippocampal/para-hippocampal gyri. }\end{array}$ \\
\hline & $\begin{array}{l}\text { Amygdala: hyperactivity at resting state; aberrant activation during fear acquisition and during the formation of abdomi- } \\
\text { nal pain-related memories. Higher FC of R amygdala positively correlated with pain intensity and decreased gut } \\
\text { permeability; FC between L amygdala, bilateral insula, and midbrain positively correlated with symptom severity. }\end{array}$ \\
\hline & $\begin{array}{l}\text { Bilateral INS: aberrant activation and FC with limbic and cortical areas at resting-state and during painful and } \\
\text { nonpainful rectal distention. R INS: aberrant activation correlated with pain intensity. L INS: abnormal activation } \\
\text { correlated with symptom severity and interoceptive awareness; aINS hyperactive during pain anticipation. }\end{array}$ \\
\hline Attentional areas & $\begin{array}{l}\text { Resting state: decreased activity of the DMN. Rectal stimulation: ACC and PFC hyperactivity. Pain anticipation: mPFC } \\
\text { and MFG aberrant activation. Painful distension: greater stress-induced activation in insula and VLPFC, and } \\
\text { hypoactivation of DLPFC and subgenual ACC. History of adverse life event was associated with altered FC in } \\
\text { the salience network and in the executive control network. Regions of the salience network (MCC, MTG, and STG) } \\
\text { were positively correlated with proinflammatory genes (IL-6 and APOL2). }\end{array}$ \\
\hline
\end{tabular}

S1, primary somatosensory cortex; M1, primary motor cortex; FC, functional connectivity; H-IBS, hypersensitive IBS; pINS, posterior insula; N-IBS, normosensitive IBS; INS, insula; R, right; L, left; aINS, anterior insula; DMN, default mode network; ACC, anterior cingulate corte; PFC, prefrontal cortex; mPFC, medial prefrontal cortex; MFG, middle frontal gyrus; VLPFC, ventrolateral prefrontal cortex; DLPFC, dorsolateral prefrontal cortex; MCC, mid-cingulate cortex; MTG, middle temporal gyrus; STG, superior temporal gyrus; APOL2, apolipoprotein L2 gene.

with anterior MCC, aINS, bilateral dorsolateral prefrontal cortex (DLPFC), and inferior parietal lobule. Pre-central cortices with stronger FCD showed increased FC with bilateral PFC.

Gupta and colleagues ${ }^{20}$ found that IBS patients presented a greater within-network connectivity than $\mathrm{HC}$ in: (1) the salience network (putamen, insula, ACC, MCC, and supramarginal gyrus); (2) the left frontal parietal network (putamen, ACC, thalamus, and frontal cortex); (3) the DMN (precuneus, inferior parietal gyrus and angular gyrus). Within IBS patients only, early adverse life events positively correlated with (1) FC within the salience network, and (2) FC within the cerebellar network for male subjects only. The same group found that regions of the salience network, including MCC, and mid and superior temporal gyrus (STG) positively correlated with pro-inflammatory genes (IL-6 and APOL2) in patients with IBS, but negatively correlated with anti-inflammatory genes (KRT8 and APOA4) in HC, suggesting that, in IBS, a peripheral pro-inflammatory state is maintained by chronically activated stress signalling pathways. ${ }^{23}$

Several studies further examined the DMN. ${ }^{24,25}$ IBS patients presented decreased DMN inter-regional FC between the precuneus and (1) the ACC, (2) the middle temporal gyrus, and (3) the medial orbital SFG. Moreover, the average DMN FC negatively correlated with IBS symptom severity. ${ }^{24}$ The DMN showed altered FC with sensorimotor regions, the right amygdala, the left orbital frontal cortex, the bilateral middle, and inferior frontal gyri, and regions deputed to pain processing (periaqueductal grey and rostral ventral medulla). Interestingly, patients with IBS only showed increased FC in the right amygdala, correlated with decreased gut permeability. $^{25}$

Two studies focused on FC in 2 main regions of interoception and emotion regulation, the insula ${ }^{26}$ and the amygdala. ${ }^{27}$ Hong et $\mathrm{al}^{26}$ found that male patients, compared to females, presented increased positive connectivity of the bilateral dorsal aINS with the $\mathrm{mPFC}$, which positively correlated with scores of visceral sensitivity; conversely, female patients had greater negative connectivity of the left dorsal aINS with the left precuneus than males, which positively correlated with symptom intensity. Moreover, diseaserelated differences in the FC between the bilateral dorsal aINS and the dorsal $\mathrm{mPFC}$ were observed in female subjects. As regards the amygdala, Qi and colleagues ${ }^{27}$ found that IBS patients, compared to controls, had: (1) higher left amygdala positive FC with right insula, midbrain, right precentral gyrus (primary motor cortex [M1]), left S1/M1, left parahippocampal gyrus, and bilateral SMA; and (2) higher right amygdala positive $\mathrm{FC}$ with the right insula, midbrain, left parahippocampal gyrus, bilateral M1, and right SMA. These results hold when controlling for anxiety and depression. Pain intensity positively correlated with the FC between the right amygdala, the right M1 and right insula; symptom severity positively 
correlated with FC between the left amygdala, bilateral insula and the midbrain, and with FC between the right amygdala and right insula. Significant clinical correlations emerged also in the study of Longarzo and colleagues, ${ }^{28}$ which showed that the FC between PCC and left supramarginal gyrus/STG negatively correlated with attitude, fear and beliefs associated with hypochondriac behavior, and that the FC between left ventral aINS and bilateral supramarginal gyrus positively correlated with interoceptive awareness, the ability to perceive signals from within the body.

Icenhour and colleagues ${ }^{29}$ made a step further by evaluating $\mathrm{FC}$ within the salience, sensorimotor and DMN in a sample of selected IBS patients with and without visceral hypersensitivity (a distinction presumably subtended by differences in the processing of visceral afferent signals), compared to HC. Hypersensitive IBS (H-IBS) patients showed increased positive FC of pregenual ACC and thalamus in the salience network and of pINS in the sensorimotor network than normosensitive IBS (N-IBS). N-IBS patients (compared to $\mathrm{H}-\mathrm{IBS}$ and $\mathrm{HC}$ ) showed less positive amygdala FC and less negative dorsal aINS FC in the DMN. DMN and sensorimotor network FC were associated with rectal perception thresholds, and FC in pINS correlated with IBS symptom severity.

Ke et $\mathrm{al}^{30}$ showed that IBS patients presented higher FC in the bilateral S1, calcarine, vermis, right thalamus, left superior parietal lobule, and less FC in the bilateral ACC and MCC, PFC, right caudate, and angular gyrus (not influenced by anxiety and depression). Correlations emerged between (1) disease duration and FC in the right $\mathrm{S} 1$ (positive correlation) and $\mathrm{FC}$ in the right anterior MCC/SMA and bilateral insula (negative correlation), (2) IBS symptoms severity and FC in the left thalamus (positive correlation) and FC in the right ventral $\mathrm{mPFC}$ and $\mathrm{MFG}$ (negative correlation), and (3) pain intensity and $\mathrm{FC}$ in the left $\mathrm{S} 1$ (positive correlation) and with $\mathrm{FC}$ in right dorsal $\mathrm{mPFC}$ and left $\mathrm{MFG}$ (negative correlation). The authors concluded that the abnormal synchronization in spontaneous brain activity may reflect symptoms manifestation.

In summary, overall, aberrant FC in IBS patients was found in the salience, sensorimotor and DMN; limbic regions (amygdala and cingulate cortex in particular) showed abnormal FC with sensorimotor, insular and frontal cortical regions. These findings were associated with severity of IBS symptoms, gut permeability, and inflammatory genes, and with the presence of adverse life events. Contradictive results emerged about the influence of anxiety and depression.

\section{Functional Magnetic Resonance Imaging With Tasks}

To assess abnormalities in visceral pain processing in IBS, several studies performed functional magnetic resonance imaging (fMRI) during non-painful and painful rectal distension. Overall, patients with IBS rate rectal distension as more painful than $\mathrm{HC}^{31}$ ${ }^{34}$ and, when combined with painful heterotopic stimulation (eg, a foot in ice-cold water), rectal pain is significantly decreased in HC but not in patients with IBS. ${ }^{35,36}$ Rectal stimulation generally elicits in both IBS and HC the activation of ACC, PFC, insula, and thalamus, ${ }^{31,32,36,37}$ together with the cerebellum. ${ }^{36-39}$ Brain activation of these areas apparently increase along with rectal balloon dilation, but not in women with IBS and in patients whose symptoms lasted more than 5 years. ${ }^{40}$ More specifically, in IBS subjects, Mertz and colleagues $^{31}$ found that painful stimulation led to a greater activation in the ACC, while Yuan et $\mathrm{al}^{32}$ found it in the insula, PFC, and thalamus, suggesting a heightened pain sensitivity of the braingut axis in IBS, with a normal pattern of activation. Anxiety and depression appeared associated with subjective stimulus ratings and with cerebral activations ${ }^{34,37}$ : anxiety correlated with pain-induced activation of the right anterior MCC and pregenual ACC, and depression with pain-induced activation of left $\mathrm{PFC}$ and cerebellar areas. To further understand the role of emotional modulation of neural responses to visceral stimuli, the same group combined rectal stimulation with a psychological distraction (either stress or relaxation). ${ }^{41}$ During painful distension, patients with IBS showed a greater stress-induced increase in the insula and the ventrolateral prefrontal cortex (VLPFC), and a lower activation of the DLPFC and the subgenual ACC compared to $\mathrm{HC}$, suggesting that $\mathrm{HC}$ presented greater up-regulation during stress in these areas. Relaxation brought to a reduced modulation of the activation in the right insula, the cerebellum, the putamen, and temporal regions during non-painful distensions, and in the VLPFC, precuneus and thalamus during painful distensions. However, when controlling for levels of anxiety, only activations in the cerebellum and precuneus survived the correction. ${ }^{41}$ Similarly, Rosenberger et $\mathrm{al}^{38}$ found that in IBS patients, cerebellar activations induced by non-painful distension (right cerebellum) and by painful distension (intermediate cerebellum) correlated with depression, while non-painful induced cerebellar activations correlated with anxiety. Labus and colleagues ${ }^{42}$ aimed to evaluate sex- and disease-related differences in brain activation of IBS patients during an emotion recognition paradigm (fear and anger): male subjects (IBS and HC), showed greater activation than females in PFC, insula, and amygdala, as well as stronger connectivity between ACC, amygdala, and insula; female subjects 
showed stronger connectivity to and from the PFC.

Combining rectal and heterotopic stimulation (to elicit a simultaneous activation of endogenous descending pathways of nociceptive inhibition), Song et $\mathrm{al}^{36}$ found that (1) during rectal stimulation alone, $\mathrm{HC}$ showed greater activation bilaterally in the aINS, in the secondary somatosensory cortex (S2), and putamen than patients with IBS; and (2) during rectal plus heterotopic stimulation, HC showed greater activation in bilateral S1 and right STG, while IBS patients in bilateral STF and right inferior lobules.

Berman and colleagues ${ }^{33}$ added to this task the evaluation of brain changes during rectal distension anticipation, to assess anomalies in preparatory brain activity before painful pelvic visceral distension. In the anticipation phase, $\mathrm{HC}$ showed reduced activity in the insula, supragenual ACC, amygdala, and dorsal brainstem; IBS patients showed less anticipatory inactivation, particularly in the right pINS and dorsal brainstem; moreover, the anticipatory decrease in the pons (dorsal brainstem) was associated with higher stimulationinduced activation in right orbitofrontal areas and bilateral supragenual ACC, suggesting that deficits in preparatory inhibition of dorsal brainstem may interfere with descending corticolimbic inhibition. Expectation of pain was investigated also by Hong and colleagues, ${ }^{43}$ who performed fMRI during cued and uncued pain expectation. In absence of a cue about the occurrence of abdominal pain stimulus (ie, in front of an uncertain painful prospect), IBS subjects (especially females, compared to HC) showed greater brain activations in the affective (amygdala), interoceptive (aINS), and attentional (MFG) regions, together with the thalamus and precuneus. The authors interpreted this hyperactivation as a possible evidence that IBS subjects tend to overestimate the likelihood of painful somatic occurrence and engage brain networks involved in affective and sensory processing. Similarly, Kano and colleagues ${ }^{44}$ found that IBS patients, compared to $\mathrm{HC}$, in a condition of uncertain anticipation of painful stimulation showed greater activation of anterior MCC, thalamus, and visual processing areas, and a higher activity in PCC/MCC and precuneus induced by the following rectal distension; if, after the uncertain cue, rectal distention was not performed, IBS patients, contrary to HC, lacked to show bilateral insula activation (but the authors note how this effect might have been due to the elevated bilateral insular responses during non-distention after the safe cue, the comparison condition). The authors conclude that cue-dependent alterations in brain responses may underlie hypervigilance to visceral sensations in IBS patients. To investigate the learning processes shaping the expectation of pain in IBS, Icenhour et $\mathrm{al}^{45}$ performed $\mathrm{fMRI}$ during the formation, extinction, and reactivation of abdominal pain-related memories in
IBS patients and $\mathrm{HC}$, through a fear conditioning paradigm. IBS patients showed: (1) during the fear acquisition phase, differential activation of PFC, amygdala, and cerebellum ${ }^{34}$; (2) during extinction, enhanced differential cingulate activation; and (3) during reinstatement, greater differential hippocampal activation. ${ }^{45}$ These results shed another light on the factors that could play a role in the pathophysiology of chronic abdominal pain and suggest that extinction-based interventions might have therapeutic effects on IBS. As regards therapeutic interventions, in fact, Lowen and colleagues $^{46}$ showed, in a longitudinal fMRI study, that 16 sessions of hypnotherapy or educational intervention were both effective in reducing pain during rectal distention in both IBS and HC groups; after the treatment, brain response to distension was similar between IBS and HC, possibly indicating that this treatment may have normalized the anomalies in the central processing of visceral signals in IBS.

Kano et $\mathrm{al}^{47}$ made a step further by examining, during colorectal distention, brain activation via fMRI and heart rate variability. Patients with IBS, although all constipation-predominant (IBS-C), displayed blunted sympathovagal balance in response to colorectal distention compared to $\mathrm{HC}$; additionally, in the $\mathrm{HC}$ but not in the IBS group, brain activation in the right caudate, bilateral dorsolateral ACC and pregenual ACC in response to rectal distention positively correlated with baseline high frequency values.

Several studies performed the same task by dividing IBS subjects according to specific criteria. Patients with diarrheapredominant IBS, during heterotopic stimulation, showed deactivation in the right aINS; IBS-C showed hyperactivation of amygdala and hippocampus; $\mathrm{HC}$ showed deactivation of the periaqueductal grey, suggesting a different abnormal endogenous pain inhibitory mechanism. ${ }^{35}$ IBS patients with a history of sexual abuse showed hyperactivation in the left MCC and PCC, hypoactivation in the left supragenual ACC, and greater pain, than subjects who did not suffer abuse ${ }^{48}$; the authors hypothesized that adverse life events may enhance the activation of dorsal cingulate regions implicated in homeostatic afferent processing. IBS patients presenting inhibition or facilitation of the RIII nociceptive spinal reflex induced by rectal distension did not present differences in brain activity associated with non-painful distensions, while differences associated with painful distensions (in frontal areas, the MCC and aINS) were influenced by levels of anxiety, depression, and tendency of catastrophizing. ${ }^{37}$ Larsson and colleagues ${ }^{49}$ showed that H-IBS patients, during painful rectal distensions, had greater activation of insula and reduced deactivation in pregenual ACC than $\mathrm{HC}$ and patients with N-IBS (which did not differ to each other); during 
expectation of rectal distension, N-IBS presented more activation in right hippocampus than controls. Similarly, Lowen and colleagues ${ }^{50}$ compared H-IBS and N-IBS in their ability to engage endogenous pain modulation mechanism during habituation to repeated visceral painful stimuli. In the last trials of a series of rectal distension, NIBS showed hypoactivation in insula and amygdala, and H-IBS showed greater activation than N-IBS and $\mathrm{HC}$ in insula, ACC, and MCC; this suggests a lack of habituation to repeated delivery of rectal stimuli, in terms of both sensitization of sensory pathways and habituation of emotional arousal, consistent with IBS well-known gut-related hypervigilance.

Given the reported aberrant activation of the insula and of the DLPFC, Aizawa et al $^{51}$ assessed IBS patients' cognitive flexibility through the Wisconsin Card Sorting Test; they presented more perseverative errors than $\mathrm{HC}$, together with: (1) reduced activity of right DLPFC and right hippocampus, (2) increased activity of left pINS, (3) diminished FC from DLPFC to pre-SMA. Similarly, Hubbard and colleagues ${ }^{52}$ tested attentional abilities in IBS, by employing a form of flanker task during fMRI. Patients with IBS, with respect to $\mathrm{HC}$, had shorter reaction times during the alerting and orienting conditions, associated with: (1) greater MCC and insula activation, and (2) less activity in the right inferior frontal junction and SMA; also, they showed activation in the dorsal mPFC together with hypoactivation of the thalamus. This aberrant activity correlated with gastrointestinal-specific anxiety, pain catastrophizing, and symptom severity.

Only 1 study performed genetic analysis ${ }^{53}$ : via fMRI, they compared brain response during an affect-matching paradigm in IBS patients and $\mathrm{HC}$, further dividing participants according to their genotype (the $\mathrm{C} / \mathrm{C}$ genotype of the c. $-42 \mathrm{C}>\mathrm{T}$ polymorphism in serotonin (5-HT) receptor 3, associated with aberrant amygdalar activity while processing emotional faces, compared to T-carrier). The C/C genotype, in both IBS and HC, appeared to correlate with increased anxiety and amygdala responsiveness during emotional and non-emotional tasks; furthermore, the polymorphism was associated with severity of IBS symptoms, and only a few IBS subjects with the $\mathrm{C} / \mathrm{C}$ genotype presented significantly increased amygdala responses to nonemotional stimuli.

One of the main limits of the aforementioned studies is the high prevalence of female subjects. Guleria et $\mathrm{al}^{54}$ performed the same task in a group of male IBS patients and, consistently with the other studies, found that they hyperactivated the insula, MTG, and cerebellum in the left hemisphere compared to $\mathrm{HC}$, but did not activate the bilateral precuneus/superior parietal lobules. Moreover, it emerged that patients with IBS-C activated the right MCC while patients with IBS-D activated the left inferior orbito-frontal cortex, left calcarine, and bilateral fusiform gyri. A second limit of the majority of the fMRI studies with tasks is pointed out by Wong et al, ${ }^{55}$ as they showed that performing rectal stimulation within the scanner environment significantly increases visceral, but not somatic, pain perception in both IBS patients and HC (compared to the same exam performed outside the MRI scanner).

Taken together, these results point in the direction of a higher activation of sensitive, interoceptive and frontal areas in IBS patients following painful rectal stimulation; its anticipation is linked to an aberrant activation of attentional ( $\mathrm{mPFC}$ and MFG) and affectiveinteroceptive (amygdala, aINS, and ACC) regions. Patients with IBS also resulted in more alertness and presented a reduced attentional shift, when compared to $\mathrm{HC}$, which was associated with an abnormal frontal activation. ${ }^{51,52}$

\section{Pharmacological functional magnetic resonance imag- ing studies}

To test the hypothesis that alterations in 5-HT signaling within the brain-gut axis may be implicated in IBS pathophysiology, Labus and colleagues ${ }^{1}$ examined brain responses via fMRI at the rectal distension task after performing acute tryptophan depletion (ATD) in a group of IBS patients (all female, IBS-C) and HC. In both groups, ATD resulted in increased response of an extensive brain network to balloon distension, including the amygdala and nodes of emotional arousal and homeostatic afferent networks. These findings were consistent with an ATD-mediated disinhibition of an emotional arousal network during aversive stimulation, and increased connectivity within the same network, suggesting that 5-HT dysregulation may play a role in central pain amplification and, ultimately, in IBS pathophysiology. Also alterations in corticotropin-releasing factor (CRF) signaling pathways have been implicated in IBS pathophysiology: the CRF receptor is present in many sites of the central nervous system, and is able to influence the activity of many areas, regardless of its effect on the hypothalamuspituitary-adrenal (HPA) axis. Hubbard and colleagues ${ }^{56}$ administered the selective CRF receptor 1 antagonist GW876008 or a placebo to a group of $\mathrm{HC}$ and a group of IBS patients, while performing $\mathrm{AMRI}$ during expectation of abdominal pain. During pain expectation, both IBS patients and HC who received the CRF receptor 1 antagonist showed hypoactivation in amygdala, hippocampus, insula, ACC, and orbito-medial PFC, compared to participants who received placebo. Under placebo, patients showed higher activity in the hypothalamus and locus coeruleus (noradrenergic pathway involved in arousal responses, alarm, and visceral body 
preparation reactions) than $\mathrm{HC}$, suggesting a greater baseline reactivity in patients; activity at the hypothalamic level decreases after administration of the drug only in patients having average and high levels of state anxiety. More recently, Kano and colleagues ${ }^{57}$ found in $\mathrm{HC}$, but not in patients with IBS, a negative correlation between adrenocorticotropic hormone response to administered CRF and the activity in the pregenual ACC during rectal distention; authors, therefore, hypothesized that impaired top-down inhibitory input from the pregenual ACC to the HPA-axis may lead to altered neuroendocrine and gastrointestinal responses to $\mathrm{CRF}$ in patients with IBS. In a subsequent study, ${ }^{58}$ they also found that individuals (IBS and $\mathrm{HC}$ ) with higher levels of alexithymia showed stronger adrenocorticotropic hormone responses to CRF administration, and that brain responses to rectal distention in the right insula, putamen, right pallidum, thalamus, orbital part of inferior frontal gyrus, and left inferior temporal gyrus positively associated with alexithymia scores to a greater extent in patients with IBS than in HC.

When interpreting the results of pharmacological studies, it should be considered that Lee and colleagues ${ }^{59}$ found that the placebo condition evoked more activity in affective and cognitive regions (insula, MCC, and VLPFC) in IBS patients than in HC (although their visceral placebo analgesia was comparable).

In summary, these data show, from a biochemical perspective, an important role of 5-HT in central modulation (exerted by the amygdala on the emotional arousal circuits), higher basal activity of the noradrenergic centers, and a dysregulation of the corticotropic axis in patients with IBS.

\section{Quantitative Magnetic Resonance Spectroscopy}

Bednarska and colleagues ${ }^{11}$ found that concentrations of glutamate + glutamine $(\mathrm{Glx})$ were lower in bilateral aINS of women with IBS, compared with $\mathrm{HC}$; furthermore, a lateralization pattern emerged, with the lower Glx concentrations of the right aINS being predicted by longer pain duration, and those of the left aINS being predicted by less use of adaptive pain-coping strategies. At the same time, GABA concentrations where comparable between the 2 groups, showing overall a reduced excitatory but unaltered inhibitory neurotransmitter (NT) levels in aINS in IBS patients. Niddam and colleagues ${ }^{12}$ found a reduction in Glx in IBS patients' hippocampus, with a concentration inversely related to emotional stress indicators. Furthermore, a functional lateralization was observed as glutamate concentrations in the left, but not the right hippocampus of IBS patients negatively correlated with anxiety, pain catastrophizing, and pain duration. Icenhour and colleagues ${ }^{13}$ found that IBS patients overall did not differ from $\mathrm{HC}$ with respect to $\mathrm{mPFC}$
GABA + or Glx levels; only IBS patients with higher anxiety levels showed increased GABA+ in $\mathrm{MPFC}$, together with lower FC between $\mathrm{mPFC}$ and $\mathrm{ACC}$.

In summary, these studies, although sharing the same limitations of the fMRI studies (a female-predominant sample of IBS patients), showed a reduced excitatory NT level in aINS and hippocampus, an unvaried level of inhibitory NT in aINS, and an influence of anxiety on the levels of inhibitory NT in mPFC.

\section{Positron Emission Tomography}

Nakai and colleagues ${ }^{60}$ sought for disease-related and genderrelated effect in HT synthesis, and found that female patients with IBS, compared to $\mathrm{HC}$, presented a greater 5-HT synthesis in the right medial temporal gyrus, a multimodal sensory association cortex, widely connected to the PFC and to the limbic system through the cingulate gyrus, parahippocampus and insula. ${ }^{61-62}$ To investigate the noradrenergic activity in a sample of IBS patients, compared to HC, Berman et $\mathrm{al}^{63}$ performed double-blind study where participants had to perform an auditory vigilance task after intake of the $\alpha 2$-adrenoreceptor ( $\alpha 2 \mathrm{AR}$ ) antagonist yohimbine $(\mathrm{YOH})$, the $\alpha 2 \mathrm{AR}$ agonist clonidine, or placebo. The $\alpha 2 \mathrm{AR}$ is a presynaptic receptor that controls the release of catecholamine from the synaptic terminal: agonists like clonidine inhibit the release of norepinephrine, while antagonists like $\mathrm{YOH}$ facilitates the release of catecholamine from the synapse, which ultimately leads to a reduction in cortical metabolism. ${ }^{64}$ First, they showed that IBS patients had higher plasma noradrenergic levels than HC before and after ingestion of all drugs. Second, IBS patients showed less $\mathrm{YOH}$-mediated reduction of activity in the central arousal circuit, consistent with a downregulation (ie, fewer functional presynaptic $\alpha 2 \mathrm{AR})$. YOH-mediated reduction of activity in the brainstem and amygdala negatively associated with adverse early life event. Third, in $\mathrm{HC}$ only, the activation of anterior MCC negatively correlated with the activation of the amygdala and the subgenual ACC. One longitudinal positron emission tomography study which evaluated brain responses to rectal distention showed a stable activation in the central pain matrix but a decreased activity in limbic, paralimbic, and pontine regions, suggesting a habituation of visceral perception and central arousal in patients with IBS linked to repeated exposure to experimental aversive visceral stimuli. ${ }^{65}$

Overall, these data underline the role of specific NTs in central modulation in patients with IBS: 5-HT, which is shown to be increased in brain areas closely interconnected with the limbic circuit especially in women; norepinephrine, which seems to subtend a greater basal activity in the arousal circuits in IBS patients, espe- 
cially in those with a history of early emotional trauma.

\section{Discussion}

This study aims at reviewing the literature on functional neuroimaging in IBS patients. Most of the studies included suggested an involvement of specific areas of the brain (amygdala, insula, prefrontal, and cingulate cortex) in the pathophysiology of IBS, although many studies shared the limitations of female predominance and the presence of confounding factors (such as psychiatric comorbidities).

A first group of studies analysed brain activation and $\mathrm{FC}$ at resting-state via $\mathrm{fMRI}$. They highlighted an aberrant activation and FC of the insular, cingulate and frontal cortices, the amygdala, and the hippocampus, suggesting an altered activity of the homeostatic and salience network and of the autonomous nervous system (possibly accounting, respectively, for IBS patients' hypervigilance and HPA-axis dysregulation). In parallel, hypoactivity in the DMN emerged at the resting state: since the DMN is the brain network presenting the highest activity in the idling brain, its hypoactivity in IBS patients was interpreted as a further hint of their continuous hypervigilance and spontaneous perception of pain. ${ }^{18}$

Most studies implemented fMRI while the experimental groups were performing different tasks, with a particular focus on painful and non-painful rectal distention. Rectal stimulation seems to elicit in both IBS patients and HC the activation of ACC, PFC, insula, thalamus, and cerebellum ${ }^{31,32,36-39}$; this activation seems higher in IBS patients, ${ }^{31,32}$ as confirmed by a recent metaanalysis. ${ }^{66}$ Aberrant activation of attentional (mPFC and MFG) and affective-interoceptive (amygdala, aINS, and ACC) regions, as well as of the dorsal brainstem, are reported during anticipation of pain $^{33,43,44,65}$ and during the learning process shaping the expectation of pain, ${ }^{39,45}$ accounting, again, for an alteration in hypervigilance and in descending corticolimbic inhibition of pain-further underlined by patients' enhanced activity of noradrenergic centres. ${ }^{56,64}$ Several studies showed similarities between dysfunctional cerebral activation of IBS patients and patients with other functional disorders, such as functional dyspepsia, ${ }^{67}$ fibromyalgia, ${ }^{14,68}$ and other conditions characterized by chronic pain, such as endometriosis. ${ }^{69}$ Here, we suggest that similarities with the neural activity of patients with functional neurological disorders, FMD in particular, should be also highlighted. In a recent study, ${ }^{70}$ our group reviewed the literature on functional neuroimaging in patients with FMD: we found a decreased activation in the primary motor cortex contralateral to the symptoms and in the parietal lobe, an aberrant activation of the amygdala, and an increased temporo-parietal junction activity. Most importantly, functional connectivity findings highlighted aberrant connections between the amygdala and motor areas (including the SMA, involved in motor programming), the temporo-parietal junction and the insula. We propose a model where amygdala functional alterations have a crucial role in producing and maintaining FMD, and the aberrant FC between amygdala and the aforementioned brain regions may explain specific secondary co-causative etiopathogenetic FMD mechanisms, respectively: (1) the impaired motor conceptualization, motor preparation and the inhibition of motor execution; (2) the altered sense of agency; and (3) the deficit in processing of the affected body parts at a cognitive level. Here, as well, a predominant role of the amygdala in maintaining IBS symptoms may be highlighted, given its aberrant activation in anticipation $^{33,43,44,65}$ and regulation ${ }^{11,56,60}$ of pain. In IBS, a major role of the insula (recognized as site of self-awareness, interoception, self-regulation of emotions and of integration between emotion and motor control), must be acknowledged: bilateral insula showed aberrant activation and FC with limbic and cortical areas at restingstate $^{26,30,45}$ and during painful and nonpainful rectal distention exam (see above). Moreover, a lateralization pattern emerged, with right insular aberrant activation often correlating with pain intensity, ${ }^{27}$ and left insular abnormal activation correlating with symptom severity ${ }^{26,27}$ and levels of interoceptive awareness. ${ }^{28}$ The activity of insula in FMD was less studied, although first evidence suggests that the insula is aberrantly activated at resting-state ${ }^{71}$ and when the patient imagines or tries to move the paralyzed leg, ${ }^{72}$ and hypoactivated during emotional tasks. ${ }^{73}$ Overall, these findings suggest that the aberrant $\mathrm{FC}$ between amygdala and the insula may play a role in the production of IBS symptoms, while the aberrant FC between amygdala and the motor cortices may play a role in the production of functional motor symptoms. Moreover, the insula seems to have a role in Psychogenic Non-Epileptic Seizure (PNES - another widespread subtype of functional neurological disorder): in fact, in patients with PNES, the insula was found to be hyperactivated and its FC with motor areas to be greatly aberrant, suggesting that an aberrant insular-motor interaction may account for some psychological features underlying PNES such as dissociation. ${ }^{74}$

At the NT level, qMRS studies highlighted a dysregulation in the glutamatergic system of patients with functional disorders. Reduced Glx levels in aINS and hippocampus, unvaried levels of GABA + in aINS, and an influence of anxiety in GABA+ levels in $\mathrm{mPFC}$ were found in IBS patients. ${ }^{11-13}$ Conversely, an increase of Glx in the limbic system has been found in patients with FMD, ${ }^{15}$ in the somatosensory cortex of patients with functional dyspepsia, ${ }^{67}$ 
and in the pINS of patients with fibromyalgia. ${ }^{14}$ FMD patients also showed lower cerebrospinal fluid levels of glutamate than HC, suggesting that a glutamatergic dysfunction plays a role in FMD pathophysiology. $^{75}$

HPA axis dysregulation, widely documented in IBS patients, has been reported in FMD patients as well, ${ }^{76}$ although future studies should further investigate this area.

Finally, most of the studies included in this review corrected their results for the levels of anxious-depressive symptomatology, ${ }^{13,17,18,21}$ usually assessed through self-report questionnaires. At the resting state, several studies reported that anxiety and depression influenced the activity and $\mathrm{FC}$ of the ACC $\mathrm{AC}^{13,18,21,34,35}$ involved in affective processing, ${ }^{18}$ but not amygdala activity and $\mathrm{FC}{ }^{27}$ During painful rectal distension, the differences between IBS and HC in frontal areas, the MCC and aINS were found to be influenced by levels of anxiety, depression, and tendency of catastrophizing. ${ }^{37}$ Moreover, anxiety correlated with pain-induced activation of the right anterior MCC and pregenual ACC, and depression with pain-induced activation of left $\mathrm{PFC}$ and cerebellar areas. ${ }^{34,37}$ When combining rectal stimulation and a psychological distraction (a task implemented to understand the role of emotional modulation of neural responses to visceral stimuli), Elsenbruch and colleagues ${ }^{41}$ found that during painful distension, IBS patients showed a greater stress-induced increase in the insula and the VLPFC, and a lower activation of the DLPFC and the subgenual ACC compared to $\mathrm{HC}$, suggesting that $\mathrm{HC}$ presented greater up-regulation during stress in these areas. ${ }^{41}$

In the attempt to find a unified theoretical approach for functional disorders, recent advances in cognitive neuroscience and neurobiology in a hierarchical Bayesian framework may help. According to this approach, perception arises from the comparison of a knowledge-driven probabilistic inference on the external causes of sensory signals (top-down generative models) and the correspondent bottom-up signals which convey eventual error to the aforementioned predictions (prediction errors). ${ }^{77}$ Through prediction errors, probabilistic predictive models are re-computed and refined, in consonance with Bayesian principles; otherwise, through active inference, agents suppress prediction errors by performing actions to modify the environment and make it fit with its predictions. In fact, according to the "Free Energy Principle", each organism tries to minimize the differences between their predicted models of the environment (priors) and the sensations conveyed by their sense organs. ${ }^{78}$ This comparison results in a posterior distribution corresponding to the percept (posterior belief). This framework was applied also to psychosomatic medicine. Edwards and colleagues ${ }^{79}$ recently proposed that functional motor and sensory symptoms arise because of the presence of an abnormal prior belief at an intermediate level in the cortical hierarchy. This belief is granted too much precision through an abnormal allocation of attention, and inevitably bias the outcome of the entire process towards itself, regardless the bottom-up input. In functional sensory symptoms, an abnormal prior in sensory cortices would predict, for example, anaesthesia or pain; in functional motor symptoms, an aberrant prior in SMA would imply a wrong expectation about the proprioceptive consequence of a movement which, in a higher level of the hierarchy (eg, pre-SMA), would be explained as a failure to realize that the movement was intended (active inference). Similarly, it has been proposed ${ }^{80}$ that an abnormal prior in the control regions of the intestines, highly precise, may produce a perception of intestine pain and discomfort, although intestines sensory signals are normal. Alterations of motility of the intestine, resulting in alterations in defecation, may be a strategy to minimize prediction error.

The results of our systematic review fit perfectly within this framework (Fig. 2). The aberrant frontal activation shown in patients with FMD and with IBS may account for the abnormal attention allocated to the aberrant priors. These priors may be located in SMA in FMD, and in the insula and S2 in IBS. ${ }^{79,81}$ Anterior INS has been hypothesized by several authors as a possible neural substrate for the comparator system between predictions and errors in the interoceptive domain. ${ }^{82,83}$ Finally, the amygdala may have a predominant role in the creation and maintenance of these priors. Edwards et $\mathrm{al}^{79}$ proposed that pathological expectations, which lead to the construction of the abnormal prior when afforded too much attention and precision, may originate through a multitude of factors, from a traumatic salient event to personal and cultural beliefs, negative effects and cognitive biases. The role of the amygdala in modulating perception and attention, in the elaboration of a perceptual stimuli with a high emotional valence ${ }^{84}$ and in its retention in memory, ${ }^{85}$ is well-known. In a predictive coding framework, it has been proposed that the amygdala, with the ACC, may work as a comparator of predictions and predictions errors, ultimately leading to dysfunctional behaviours. ${ }^{80}$ Therefore, the aberrant activation and $\mathrm{FC}$ of the amygdala in functional syndromes may explain, to a certain extent, the maintenance of abnormal priors in specific brain area, producing a continuous discrepancy in the comparison between top-down and bottom-up signals, ultimately leading to each patient specific symptomatology (either motor or sensitive-visceral). This may be supported by the findings of Icenhour and colleagues ${ }^{45}$ who showed that during the formation of abdominal pain-related memories, IBS patients presented a different activity in the amygdala, compared to $\mathrm{HC}$. 


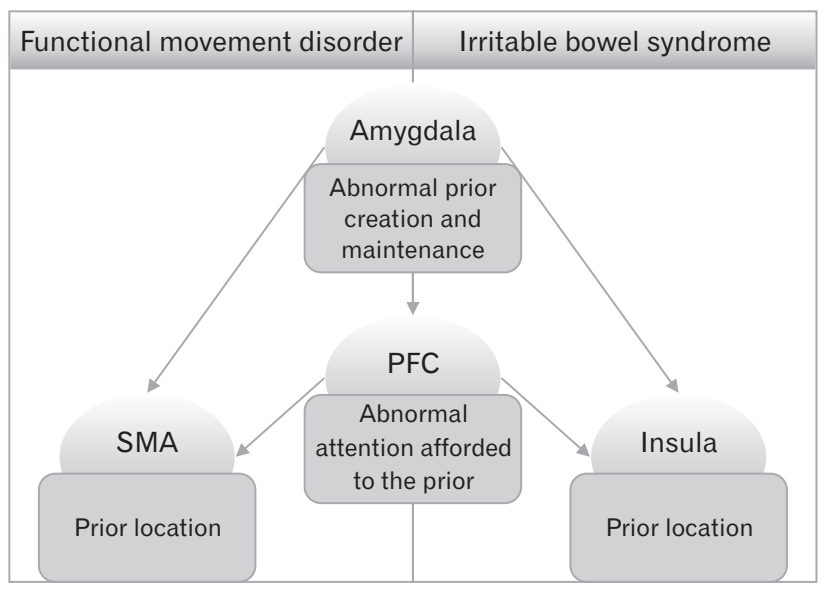

Figure 2. A common pathophysiology for irritable bowel syndrome (IBS) and functional movement disorders (FMD) in a Bayesian perspective. Recent hypotheses in psychosomatic medicine suggest that functional symptoms might arise when excessive attention is granted to abnormal prior beliefs, located at an intermediate level in the cortical hierarchy. In line with this framework, here we propose that the aberrant frontal activation shown by both patients with FMD and with IBS may account for the abnormal attention allocated to the aberrant priors, which may be located in supplementary motor area (SMA) in FMD, and in the insula and secondary somatosensory cortex (S2) in IBS. ${ }^{79,81}$ Anterior insula has been hypothesized by several authors as a possible neural substrate for the comparator system between predictions and errors in the interoceptive domain. ${ }^{82,83}$ Finally, the amygdala may have a predominant role in the creation and maintenance of these priors. ${ }^{79} \mathrm{PFC}$, prefrontal cortex.

\section{Limitations and Future Perspectives}

Our study has the following limitations. First, we discussed functional disorders only: it is possible that the brain alterations that we highlighted here are shared by several other organic diseases, representing either an epiphenomenon of the disorders or a contributing factor to their pathophysiology, as reported for pelvic pain associated with endometriosis. ${ }^{69}$ Second, although we mentioned other functional disorders such as functional dyspepsia, fibromyalgia and PNES, we mostly focused on FMD; next steps in the research agenda would be (1) to revise the literature about neuroimaging in other functional disorders, and (2) to conduct experimental neuroimaging studies, comparing patients with different functional symptoms, in order to investigate both their common core and their specificity. Third, we included mostly cross-sectional studies: hence, we cannot exclude the hypothesis that these common brain alterations are only common epiphenomena of the functional disorders, and we cannot confirm our hypothesis that they may account for IBS (and functional disorders) pathophysiology. Fourth, we did not conduct a meta-analysis or quantitative analysis, which would have reinforced our claim; future meta-analytic studies should assess whether this similarity is confirmed when focusing on the subregions of the aforementioned common areas.

\section{Conclusions}

Functional neuroimaging helps detecting, in patients with IBS, several brain alterations which appear very similar to the ones observed in patients with other functional disorders, such as FMD. Overall, our findings lead us to speculate, under a theoretical and neurobiological perspective, that different functional disturbances may share a common pathophysiology.

\section{Supplementary Material}

Note: To access the supplementary table mentioned in this article, visit the online version of Journal of Neurogastroenterology and Motility at http://www.jnmjournal.org/, and at https://doi. org/10.5056/jnm21079.

Acknowledgements: Aldo Ravelli Research Center partially supported the study.

\section{Financial support: None.}

\section{Conflicts of interest: None.}

Author contributions: Conception and design of the study: Veronica Nisticò, Roberta E Rossi, Andrea M D'Arrigo, and Benedetta Demartini; acquisition and analysis of data: Veronica Nisticò, Roberta E Rossi, Andrea M D'Arrigo, and Benedetta Demartini; drafting the manuscript or figures: Veronica Nisticò, Roberta $\mathrm{E}$ Rossi, and Andrea M D'Arrigo; and revising the manuscript for intellectual content: Alberto Priori, Orsola Gambini, and Benedetta Demartini.

\section{References}

1. Labus JS, Mayer EA, Jarcho J, et al. Acute tryptophan depletion alters the effective connectivity of emotional arousal circuitry during visceral stimuli in healthy women. Gut. 2011;60:1196-203.

2. Mearin F, Lacy BE, Chang L, et al. Bowel disorders. Gastroenterology 2016;150:1393-1407, e5.

3. Tanaka Y, Kanazawa M, Fukudo S, Drossman DA. Biopsychosocial model of irritable bowel syndrome. J Neurogastroenterol Motil 2011;17:131-139. 
4. Boeckxstaens G, Camilleri M, Sifrim D, et al. Fundamentals of neurogastroenterology: physiology/motility-sensation. Gastroenterology 2016;150:1292-1304, e2.

5. Vanner S, Greenwood-Van Meerveld B, Mawe G, et al. Fundamentals of neurogastroenterology: basic science. Gastroenterology 2016;150:12801291.

6. Mayer EA, Tillisch K, Gupta A. Gut/brain axis and the microbiota. J Clin Invest 2015;125:926-938.

7. Van Oudenhove L, Levy RL, Crowell MD, et al. Biopsychosocial aspects of functional gastrointestinal disorders: how central and environmental processes contribute to the development and expression of functional gastrointestinal disorders. Gastroenterology 2016;150:1355-1367.

8. Al Omran Y, Aziz Q. The brain-gut axis in health and disease. Adv Exp Med Biol 2014;817:135-153.

9. Elsenbruch S. Abdominal pain in irritable bowel syndrome: a review of putative psychological, neural and neuro-immune mechanisms. Brain Behav Immun 2011;25:386-394.

10. Mayer EA, Tillisch K. The brain-gut axis in abdominal pain syndromes. Annu Rev Med 2011;62:381396.

11. Bednarska O, Icenhour A, Tapper S, et al. Reduced excitatory neurotransmitter levels in anterior insulae are associated with abdominal pain in irritable bowel syndrome. Pain 2019;160:2004-2012.

12. Niddam DM, Tsai SY, Lu CL, Ko CW, Hsieh JC. Reduced hippocampal glutamate-glutamine levels in irritable bowel syndrome: preliminary findings using magnetic resonance spectroscopy. Am J Gastroenterol 2011;106:1503-1511.

13. Icenhour A, Tapper S, Bednarska O, et al. Elucidating the putative link between prefrontal neurotransmission, functional connectivity, and affective symptoms in irritable bowel syndrome. Sci Rep 2019;9:13590.

14. Harris RE, Sundgren PC, Craig AD, et al. Elevated insular glutamate in fibromyalgia is associated with experimental pain. Arthritis Rheum 2009;60:3146-3152.

15. Demartini B, Gambini O, Uggetti C, et al. Limbic neurochemical changes in patients with functional motor symptoms. Neurology 2019;93:e52-e58.

16. Moher D, Liberati A, Tetzlaff J, Altman DG; PRISMA Group. Preferred reporting items for systematic reviews and meta-analyses: the PRISMA statement. PloS Med 2009;6:e1000097.

17. Ma X, Li S, Tian J, et al. Altered brain spontaneous activity and connectivity network in irritable bowel syndrome patients: a resting-state fMRI study. Clin Neurophysiol 2015;126:1190-1197.

18. Qi R, Liu C, Ke J, et al. Intrinsic brain abnormalities in irritable bowel syndrome and effect of anxiety and depression. Brain Imaging Behav 2016;10:1127-1134.

19. Hong JY, Kilpatrick LA, Labus J, et al. Patients with chronic visceral pain show sex-related alterations in intrinsic oscillations of the resting brain. J Neurosci 2013;33:11994-12002.

20. Gupta A, Kilpatrick L, Labus J, et al. Early adverse life events and resting-state neural networks in patients with chronic abdominal pain: evidence for sex differences. Psychosom Med 2014;76:404-412.

21. Qi R, Liu C, Weng Y, et al. Disturbed interhemispheric functional connectivity rather than structural connectivity in irritable bowel syndrome.
Front Mol Neurosci 2016;9:141.

22. Weng Y, Qi R, Liu C, et al. Disrupted functional connectivity density in irritable bowel syndrome patients. Brain Imaging Behav 2017;11:18121822.

23. Gupta A, Cole S, Labus JS, et al. Gene expression profiles in peripheral blood mononuclear cells correlate with salience network activity in chronic visceral pain: a pilot study. Neurogastroenterol Motil 2017;29:e13027.

24. Qi R, Ke J, Schoepf UJ, et al. Topological reorganization of the default mode network in irritable bowel syndrome. Mol Neurobiol 2016;53:6585-6593.

25. Witt ST, Bednarska O, Keita $\AA$ V, et al. Interactions between gut permeability and brain structure and function in health and irritable bowel syndrome. Neuroimage Clin 2019;21:101602.

26. Hong JY, Kilpatrick LA, Labus JS, et al. Sex and disease-related alterations of anterior insula functional connectivity in chronic abdominal pain. J Neurosci. 2014;34:14252-14259.

27. Qi R, Liu C, Ke J, et al. Abnormal amygdala resting-state functional connectivity in irritable bowel syndrome. AJNR Am J Neuroradiol 2016;37:1139-1145.

28. Longarzo M, Quarantelli M, Aiello M, et al. The influence of interoceptive awareness on functional connectivity in patients with irritable bowel syndrome. Brain Imaging Behav 2017;11:1117-1128.

29. Icenhour A, Witt ST, Elsenbruch S, et al. Brain functional connectivity is associated with visceral sensitivity in women with irritable bowel syndrome. Neuroimage Clin 2017;15:449-457.

30. Ke J, Qi R, Liu C, et al. Abnormal regional homogeneity in patients with irritable bowel syndrome: a resting-state functional MRI study. Neurogastroenterol Motil 2015;27:1796-1803.

31. Mertz H, Morgan V, Tanner G, et al. Regional cerebral activation in irritable bowel syndrome and control subjects with painful and nonpainful rectal distention. Gastroenterology 2000;118:842-848.

32. Yuan YZ, Tao RJ, Xu B, et al. Functional brain imaging in irritable bowel syndrome with rectal balloon-distention by using fMRI. World J Gastroenterol 2003;9:1356-1360.

33. Berman SM, Naliboff BD, Suyenobu B, et al. Reduced brainstem inhibition during anticipated pelvic visceral pain correlates with enhanced brain response to the visceral stimulus in women with irritable bowel syndrome. J Neurosci 2008;28:349-359.

34. Elsenbruch S, Rosenberger C, Enck P, Forsting M, Schedlowski M, Gizewski ER. Affective disturbances modulate the neural processing of visceral pain stimuli in irritable bowel syndrome: an fMRI study. Gut 2010;59:489-495.

35. Wilder-Smith CH, Schindler D, Lovblad K, Redmond SM, Nirkko A. Brain functional magnetic resonance imaging of rectal pain and activation of endogenous inhibitory mechanisms in irritable bowel syndrome patient subgroups and healthy controls. Gut 2004;53:1595-1601.

36. Song GH, Venkatraman V, Ho KY, Chee MW, Yeoh KG, Wilder-Smith $\mathrm{CH}$. Cortical effects of anticipation and endogenous modulation of visceral pain assessed by functional brain MRI in irritable bowel syndrome patients and healthy controls. Pain 2006;126:79-90.

37. Bouhassira D, Moisset X, Jouet P, Duboc H, Coffin B, Sabate JM. Changes in the modulation of spinal pain processing are related to sever- 
ity in irritable bowel syndrome. Neurogastroenterol Motil 2013;25:623e468.

38. Rosenberger C, Thürling M, Forsting M, Elsenbruch S, Timmann D, Gizewski ER. Contributions of the cerebellum to disturbed central processing of visceral stimuli in irritable bowel syndrome. Cerebellum 2013;12:194-198.

39. Claassen J, Labrenz F, Ernst TM, et al. Altered cerebellar activity in visceral pain-related fear conditioning in irritable bowel syndrome. Cerebellum 2017;16:508-517.

40. Wang D, Zhang X, Zhang X, Huang Z, Song Y. Magnetic resonance imaging analysis of brain function in patients with irritable bowel syndrome. BMC Gastroenterol 2017;17:148.

41. Elsenbruch S, Rosenberger C, Bingel U, Forsting M, Schedlowski M, Gizewski ER. Patients with irritable bowel syndrome have altered emotional modulation of neural responses to visceral stimuli. Gastroenterology 2010;139:1310-1319.

42. Labus JS, Gupta A, Coveleskie K, et al. Sex differences in emotion-related cognitive processes in irritable bowel syndrome and healthy control subjects. Pain 2013;154:2088-2099.

43. Hong JY, Naliboff B, Labus JS, et al. Altered brain responses in subjects with irritable bowel syndrome during cued and uncued pain expectation. Neurogastroenterol Motil 2016;28:127-138.

44. Kano M, Muratsubaki T, Morishita J, et al. Influence of uncertain anticipation on brain responses to aversive rectal distension in patients with irritable bowel syndrome. Psychosom Med 2017;79:988-999.

45. Icenhour A, Langhorst J, Benson S, et al. Neural circuitry of abdominal pain-related fear learning and reinstatement in irritable bowel syndrome. Neurogastroenterol Motil 2015;27:114-127.

46. Lowén MB, Mayer EA, Sjöberg M, et al. Effect of hypnotherapy and educational intervention on brain response to visceral stimulus in the irritable bowel syndrome. Aliment Pharmacol Ther 2013;37:1184-1197.

47. Kano M, Yoshizawa M, Kono K, et al. Parasympathetic activity correlates with subjective and brain responses to rectal distension in healthy subjects but not in non-constipated patients with irritable bowel syndrome. Sci Rep 2019;9:7358.

48. Ringel Y, Drossman DA, Leserman JL, et al. Effect of abuse history on pain reports and brain responses to aversive visceral stimulation: an FMRI study. Gastroenterology 2008;134:396-404.

49. Larsson MB, Tillisch K, Craig AD, et al. Brain responses to visceral stimuli reflect visceral sensitivity thresholds in patients with irritable bowel syndrome. Gastroenterology 2012;142:463-472, e3.

50. Lowén MB, Mayer E, Tillisch K, et al. Deficient habituation to repeated rectal distensions in irritable bowel syndrome patients with visceral hypersensitivity. Neurogastroenterol Motil 2015;27:646-655.

51. Aizawa E, Sato Y, Kochiyama T, et al. Altered cognitive function of prefrontal cortex during error feedback in patients with irritable bowel syndrome, based on FMRI and dynamic causal modeling. Gastroenterology 2012;143:1188-1198.

52. Hubbard CS, Hong J, Jiang Z, et al. Increased attentional network functioning related to symptom severity measures in females with irritable bowel syndrome. Neurogastroenterol Motil 2015;27:1282-1294.

53. Kilpatrick LA, Labus JS, Coveleskie K, et al. The HTR3A polymor- phism c. $-42 \mathrm{C}>\mathrm{T}$ is associated with amygdala responsiveness in patients with irritable bowel syndrome. Gastroenterology 2011;140:1943-1951.

54. Guleria A, Karyampudi A, Singh R, et al. Mapping of brain activations to rectal balloon distension stimuli in male patients with irritable bowel syndrome using functional magnetic resonance imaging. J Neurogastroenterol Motil 2017;23:415-427.

55. Wong RK, Van Oudenhove L, Li X, Cao Y, Ho KY, Wilder-Smith CH. Visceral pain perception in patients with irritable bowel syndrome and healthy volunteers is affected by the MRI scanner environment. United European Gastroenterol J 2016;4:132-141.

56. Hubbard CS, Labus JS, Bueller J, et al. Corticotropin-releasing factor receptor 1 antagonist alters regional activation and effective connectivity in an emotional-arousal circuit during expectation of abdominal pain. $\mathrm{J}$ Neurosci 2011;31:12491-12500

57. Kano M, Muratsubaki T, Van Oudenhove L, et al. Altered brain and gut responses to corticotropin-releasing hormone $(\mathrm{CRH})$ in patients with irritable bowel syndrome. Sci Rep 2017;7:12425.

58. Kano M, Muratsubaki T, Yagihashi M, et al. Insula activity to visceral stimulation and endocrine stress responses as associated with alexithymia in patients with irritable bowel syndrome. Psychosom Med 2020;82:2938.

59. Lee HF, Hsieh JC, Lu CL, et al. Enhanced affect/cognition-related brain responses during visceral placebo analgesia in irritable bowel syndrome patients. Pain 2012;153:1301-1310.

60. Nakai A, Kumakura Y, Boivin M, et al. Sex differences of brain serotonin synthesis in patients with irritable bowel syndrome using alpha-[11C] methyl-L-tryptophan, positron emission tomography and statistical parametric mapping. Can J Gastroenterol 2003;17:191-196.

61. Nieuwenhuys R, Voogd J, van Huijzen C. The human central nervous system, 3rd ed. New York: Springer-Verlag Inc, 1988.

62. Mesulam MM. From sensation to cognition. Brain 1998;121(pt 6):1013-1052.

63. Berman S, Suyenobu B, Naliboff BD, et al. Evidence for alterations in central noradrenergic signaling in irritable bowel syndrome. Neuroimage 2012;63:1854-1863

64. Bremner JD, Innis RB, $\mathrm{Ng} \mathrm{CK}$, et al. Positron emission tomography measurement of cerebral metabolic correlates of yohimbine administration in combat-related posttraumatic stress disorder. Arch Gen Psychiatry 1997;54:246-254.

65. Naliboff BD, Berman S, Suyenobu B, et al. Longitudinal change in perceptual and brain activation response to visceral stimuli in irritable bowel syndrome patients. Gastroenterology 2006;131:352-365.

66. Tillisch K, Mayer EA, Labus JS. Quantitative meta-analysis identifies brain regions activated during rectal distension in irritable bowel syndrome. Gastroenterology 2011;140:91-100.

67. Mak AD, Northoff G, Yeung DK, et al. Increased glutamate in somatosensory cortex in functional dyspepsia. Sci Rep 2017;7:3926.

68. De Paepe B, Smet J, Baeken C, Van Oosterwijck J, Meeus M. A capital role for the brain's insula in the diverse fibromyalgia-associated symptoms. Med Hypotheses 2020;143:110077.

69. As-Sanie S, Kim J, Schmidt-Wilcke T, et al. Functional connectivity is associated with altered brain chemistry in women with endometriosis- 
associated chronic pelvic pain. J Pain 2016;17:1-13.

70. Demartini B, Nisticò V, Edwards MJ, Gambini O, Priori A. The pathophysiology of functional movement disorders. Neurosci Biobehav Rev 2021;120:387-4500.

71. Stone J, Zeman A, Simonotto E, et al. FMRI in patients with motor conversion symptoms and controls with simulated weakness. Psychosom Med 2007;69:961-969.

72. Saj A, Raz N, Levin N, Ben-Hur T, Arzy S. Disturbed mental imagery of affected body-parts in patients with hysterical conversion paraplegia correlates with pathological limbic activity. Brain Sci 2014;4:396-404.

73. Espay AJ, Maloney T, Vannest J, et al. Dysfunction in emotion processing underlies functional (psychogenic) dystonia. Mov Disord 2018;33:136145.

74. Mcsweeney M, Reuber M, Levita L. Neuroimaging studies in patients with psychogenic non-epileptic seizures: a systematic meta-review. Neuroimage Clin 2017;16:210-221.

75. Demartini B, Invernizzi RW, Campiglio L, et al. Cerebrospinal fluid glutamate changes in functional movement disorders. NPJ Parkinsons Dis 2020;6:37.

76. Apazoglou K, Mazzola V, Wegrzyk J, Frasca Polara G, Aybek S. Biological and perceived stress in motor functional neurological disorders.
Psychoneuroendocrinology 2017;85:142-150.

77. Clark A. Whatever next? Predictive brains, situated agents, and the future of cognitive science. Behav Brain Sci 2013;36:181-204.

78. Friston K. The free-energy principle: a rough guide to the brain? Trends Cogn Sci 2009;13:293-301.

79. Edwards MJ, Adams RA, Brown H, Pareés I, Friston KJ. A Bayesian account of 'hysteria'. Brain 2012;135(pt 11):3495-3512.

80. Ohira H. Regulation of functions of the brain and body by the principle of predictive coding: implications for Impairments of the brain-gut axis. Psychological Topics 2018;27:1-15.

81. Wiech K, Ploner M, Tracey I. Neurocognitive aspects of pain perception. Trends Cogn Sci 2008;12:306-313.

82. Seth AK. Interoceptive inference, emotion, and the embodied self. Trends Cogn Sci 2013; 17:565-573.

83. Seth AK, Critchley HD. Extending predictive processing to the body: emotion as interoceptive inference. Behav Brain Sci 2013;36:227-228.

84. Vuilleumier P. The role of the human amygdala in perception and attention. The Guilford Press. 2009.

85. McGaugh JL. The amygdala modulates the consolidation of memories of emotionally arousing experiences. Annu Rev Neurosci 2004;27:1-28. 